\title{
BMJ Open International normative data for paediatric foot posture assessment: a cross-sectional investigation
}

\author{
Gabriel Gijon-Nogueron, ${ }^{1,2}$ Alfonso Martinez-Nova, ${ }^{3}$ Pilar Alfageme-Garcia, ${ }^{3}$ \\ Jesus Montes-Alguacil, ${ }^{1}$ Angela Margaret Evans ${ }^{4}$
}

To cite: Gijon-Nogueron G, Martinez-Nova A, AlfagemeGarcia P, et al. International normative data for paediatric foot posture assessment: a cross-sectional investigation. BMJ Open 2019;9:e023341. doi:10.1136/ bmjopen-2018-023341

\section{- Prepublication history for} this paper is available online. To view these files, please visit the journal online (http://dx.doi. org/10.1136/bmjopen-2018023341).

GG-N and AM-N contributed equally.

Received 4 April 2018 Revised 21 February 2019 Accepted 26 February 2019

\section{Check for updates}

(c) Author(s) (or their employer(s)) 2019. Re-use permitted under CC BY-NC. No commercial re-use. See rights and permissions. Published by BMJ.

${ }^{1}$ Nursing and Podiatry, Universidad de Malaga, Malaga, Spain

${ }^{2}$ Instituto de Investigación Biomédica de Málaga (IBIMA),

Malaga, Spain

${ }^{3}$ Nursing and Podiatry, Universidad de Extremadura, Caceres, Spain

${ }^{4}$ Podiatry, La Trobe University College of Science Health and Engineering, Bundoora, Victoria, Australia

Correspondence to Dr Gabriel Gijon-Nogueron; gagijon@uma.es

\begin{abstract}
Objectives The foot posture index (FPI) is an observational tool designed to measure the position of the foot. The objective of this study was to establish international reference data for foot posture across childhood, and influence of body mass index (BMI) on paediatric foot posture.

Design Cross-sectional study.

Setting and participants The dataset comprised 3217 healthy children, aged from 3 to 15 years. Contributing data were acquired from Spain, UK and Australia. Interventions Foot posture was described by means and z-score of the FPI and the height and weight of each subject was measured and the BMI was calculated.

Results The foot posture of 3217 children were reviewed. A pronated (FPI $\geq+6$ ) foot posture was found in 960 $(29.8 \%)$ children, a normal (FPI 0 to +6 ) foot posture in $1776(55.2 \%)$ and a highly pronated (FPI +10$)$ foot posture was found in 127 children (3.9\%) (range -4 to $+12 \mathrm{FPI}$ ). Less than $11 \%$ were found to have a supinated foot type $(n=354)$. Approximately $20 \%$ of children were overweight/ obese, but correlation between BMI and FPI was weak and inverse $(r=-0.066, p<0.01)$, refuting the relationship between increased body mass and flatfeet.

Conclusions This study confirms that the 'flat' or pronated foot is the common foot posture of childhood, with FPI score of +4 (3) the average finding. Trend indicated a less flatfoot with age, although non-linear. A wide normal range of foot posture across childhood is confirmed.
\end{abstract}

\section{INTRODUCTION}

Paediatric foot posture is a common parental concern, a frequent presentation to clinicians and an area of dispute regarding both the need for treatment. ${ }^{12}$ The term 'flatfoot' has referred to a foot which is nearly or completely contacting the ground, ${ }^{3}$ and has been evaluated using foot posture, ${ }^{4}$ footprints, ${ }^{5}$ radiological and anthropometric measures $^{6}$ and is often poorly defined. ${ }^{7}$ From a clinical practice perspective, there is no single universally accepted diagnostic technique.

A systematic review ${ }^{8}$ has addressed flatfoot and clinical measures, in healthy

\section{Strengths and limitations of this study}

First study to measure foot posture with any method in a sample of 3217 children.

- Comparison between different countries strengthens the study findings.

- The sample does not balance the number of children from each country.

- The disproportionate numbers of children within each age year group.

children, finding flatfoot the expected foot posture before 8 years of age, due to young osseous structures, ligament laxity, increased adipose tissue and immature neuromuscular control. $^{910}$ With variation, flatfoot posture reduces across a child's first 10 years. ${ }^{11-13}$ Some children with flexible flatfeet experience lower limb pain ${ }^{14}$ with compromised gait. ${ }^{15}$ The quandary for clinicians is discerning when a child's foot is within or outside the developmental range, so that parents may be reassured, advised to monitor with growth or to treat. ${ }^{1617}$

Children's foot posture has been interpreted with footprint assessments in many studies, with inference of a problematic flatfoot when the footprint area is increased. Throughout early childhood, children continue to develop a skeletal medial longitudinal foot arch,$^{18}$ different from the adult population $^{19}$ and altered children's foot posture must be evaluated in context of developmental stage and the presence/absence of systemic influences, such as hypotonia and hypermobility, which may be non-specific or syndromic, for example, Down or Marfan's syndromes.

Despite the fact that paediatric flatfoot is a frequent concern, ${ }^{120}$ the evidence for treatment is weak. The lack of definition for flatfoot has contributed to varying opinions and a lack of consensus for best practice..$^{21-23}$ There is no 'gold standard' for categorising 
foot type, with the margins of flat/rectus/high arch often undefined. Further, few clinical measures are validated in children; hence, it is common for clinicians to make diagnostic decisions based on their personal clinical experience of foot types. ${ }^{24}$ The availability of normal reference data for paediatric foot posture, based on a valid measure, will provide a 'benchmark' for clinical evaluation of this frequent clinical concern. The paediatric flatfoot proforma (p-FFP) has attempted to standardise diagnoses, and direct when intervention is required, using a combination of subjective assessment points and a range of foot posture measures. ${ }^{17}$ However, the extent to which the p-FFP is used by clinicians is unknown and the proforma does not specify management techniques.

Ten years ago, the foot posture index (FPI-6) ${ }^{25}$ emerged with the objective to standardise the assessment of foot posture in stance across three discrete foot regions (rearfoot, midfoot, forefoot) enabling feet to be scored and categorised. Both Evans and Karimi ${ }^{26}$ and Gijon-Nogueron $e t a l^{27}$ have previously utilised the FPI to investigate the paediatric foot. The FPI is a quick, and easy-to-use clinical tool, not requiring equipment. Scrutiny of the FPI demonstrates it repeatable and valid, ${ }^{4}$ with excellent interrater reliability in assessment of the paediatric foot. ${ }^{28}$ Recently, the FPI has been identified as a preferred method of paediatric foot posture measurement in future research. $^{29}$

\section{METHODS \\ Data acquisition}

Data were acquired from multiple sources where the FPI had been assessed in different children, recruited for screening studies or acting as comparative controls. Three datasets were acquired from the authors' previous works $(\mathrm{n}=1032, \mathrm{n}=1457, \mathrm{n}=728) .{ }^{19} 2627$ Measurements were taken during 2010 and 2016, some from 10 schools randomly selected from 25 schools located in the provinces of Málaga, Granada and Plasencia (Spain) $(\mathrm{n}=2489)$. In the UK $(n=225)$ and Australia $(n=503)$, two datasets were acquired from the author's previous works investigating the reliability of clinical assessment measures $(\mathrm{n}=170),{ }^{10}{ }^{30}$ and further datasets were acquired from other authors in the UK investigating foot posture in young children $(\mathrm{n}=225),{ }^{31}$ and Australia investigating foot posture in young children with Sever's disease $(n=303),{ }^{32}$ and the control group from an idiopathic toe-walking study $(n=30) \cdot{ }^{33}$ A total of 3217 observations of the FPI in children aged from 3 to 15 years were collated.

\section{Participants}

The inclusion criterion across the studies was for children, of both genders, and aged between 3 and 15 years. Exclusion criteria were foot pain at the time of examination, history of injury to the lower limbs (eg, musculoskeletal injuries during the previous 6 months), congenital foot abnormalities, cerebral palsy, motor dysfunction, inflammatory disorders or foot surgery.

\section{Protocol}

Foot posture was assessed with all subjects barefoot, in a relaxed standing position using the standard protocol for the FPI. ${ }^{34}$ The FPI evaluates the multisegmental nature of foot posture in all three planes, and does not require the use of specialised equipment. Each item of the FPI is scored between -2 and +2 , with the total six items referring to positions of the forefoot, midfoot and hindfoot, and the three planes of motion: (1) talar head palpation; (2) symmetry of supra and infra lateral malleolar curvature; (3) inversion/eversion of the calcaneus; (4) prominence in the region of the talonavicular joint; (5) height of the medial longitudinal arch; (6) abduction/ adduction of the forefoot. The FPI score may range from -12 (highly supinated) to +12 (highly pronated). The statistical analysis was independent of the outcome assessors. The FPI assessors were blinded with the data passed directly to the database for entry and analyses. Good interobserver reliability was recorded (Intraclass correlation (ICC) 0.852-0.895) across the studies.

The body mass index (BMI) was calculated from the children's height and weight, calculated as, BMI=weight $(\mathrm{kg}) /$ height $(\mathrm{m})^{2}$. In Spain, the Orbegozo ${ }^{35}$ BMI classification is used; in Australia, the Australian Health Survey $^{36}$; and in UK, the National Child Measurement Programme. ${ }^{37}$ Accordingly, we classified children by their BMI score, using the systems proposed by Orbegozo, Australian Health Survey and Public Health England, allocating children to one of four categories: underweightpercentile $<3$ (, normal weight-percentile between 3 and 90 , overweight-percentile between 90 and 97 and obesity-percentile $>97$, based on BMI z-score, and age.

\section{Patient and public involvement}

No patients were involved in setting the research question or the outcome measures, nor were they involved in the design or conduct of the study. No patients were asked to advise on interpretation or writing up of results. There are no plans to disseminate the results of the research to study participants.

\section{Data analysis}

Data were entered and all analyses were performed using constructed datasets in SPSS V.24 software packages. The data from the contributing studies were collated in a separate database, with statistical analysis performed by an external person, previously blind to the results.

Testing for normality using a Kolmogorov-Smirnov test, found non-normal distribution of all data, indicating suitability for non-parametric analysis (Mann-Whitney U and Kruskal-Wallis). Descriptive statistics (mean, SD, minimum, maximum, frequencies) were used to examine the basic anthropometrical characteristics of the study populations. The FPI was analysed as continuous data, rather than as z-score data, and analysis of variance was conducted to determine the association between the different BMI groups (underweight, normal, overweight and obesity), gender, age and the FPI. To preserve the 


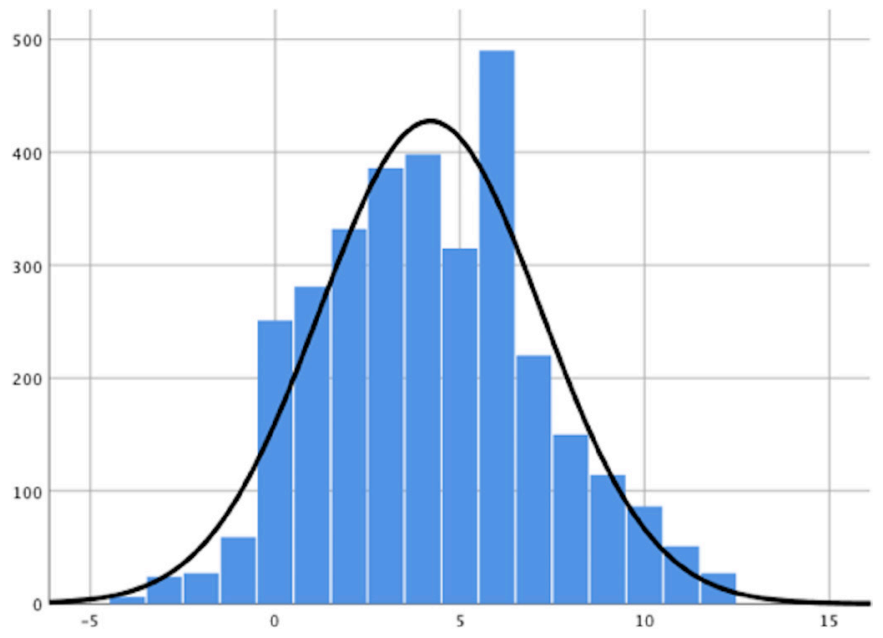

FPI Score

Figure 1 Frequency plot of FPI values $(n=3217)$. FPI, foot posture index.

independence of data, ${ }^{25}$ and based on the strong correlation between FPI scores for left and right feet, ${ }^{38}$ only the left foot (chosen at random) was used in the statistical analyses, applying the Games-Howell post hoc correction to identify significant differences. With reference to the available normative data, ${ }^{25}$ three FPI- 6 scores levels were used to 'define and explore' the range supinated ( -12 to $-1)$, neutral $(0$ to +5$)$, pronated $(+6$ to +8$)$ and overpronated $(+9$ to +12$)$. The significance level was set at $\mathrm{p}<0.05$, and all the analyses and tests were two-sided.

\section{RESULTS}

The mean age of the study population of 3217 children was 8.67 years (SD 2.02), ranging from 3 to 15 years. The mean BMI was $19.08 \mathrm{~kg} / \mathrm{m}^{2}$ (SD 4.05), ranging from 10.57 to $39.14 \mathrm{~kg} / \mathrm{m}^{2}$. The mean FPI score was 4.11 (SD 2.92) and 4.20 (3.00) for left and right feet, respectively, with whole scores ranging from -4 to +12 (left and right) (figure 1, FPI right feet). The total study population gender distribution was 1699 male and 1518 female (table 1).

In the study population of 3217 children, flatfeet or pronated (FPI $\geq+6)$ were found in $960(29.8 \%)$ children and normal (FPI $<+6)$ in $1776(55.2 \%)$ children, FPI $\geq+10$ yielded flatfeet in $127(3.9 \%)$ cases and supinated foot were found in $354(11 \%)$ children. Table 2 used designated FPI categories to define and explore the range of foot posture across childhood.

There was strong correlation between FPI scores on left and right sides $(\mathrm{r}=0.9014, \mathrm{p}<0.01)$, from which the left side was arbitrarily used for subsequent analyses. Similarly, we found little gender bias, with the mean FPI for males 4.2 (2.9), range -4 to +12 , and for females, mean FPI 3.99 (2.9), range -4 to +12 (figure 2 ). The correlation between FPI and gender used z-score was very weak, if significant $(\mathrm{F}=4.073, \mathrm{p}=0.04)$. Between countries there was significant difference $(\mathrm{p}<0.01)$, with Spanish children's mean FPI $=4.00$ (2.9), the UK mean FPI $=4.9$ (3.3) and the Australian children's mean FPI=4.7 (3.1).

The general trend showed FPI scores declining with age, which supports the clinical observation of less flatfoot in older children. The frequency of FPI scores for each year of age show that the maximum FPI score $=+6$, in $15.2 \%$ of children (table 3 ).

Clinical alert is indicated for foot posture $> \pm 2 \mathrm{SD}$, representing $5 \%$ of expected abnormality. Table 4 shows the SD of the mean FPI scores and enables the mean FPI and 1 and 2 SD above and below to be referenced as normally expected for each year of age. Figure 3 displays and explores the relationship between foot posture and age across childhood for the study population, using error bars for average z-scores (95\% CIs).

Significant correlation was found between BMI and age $(\mathrm{r}=0.276, \mathrm{p}<0.01)$. The correlation between BMI and FPI, while also statistically significant, was very weak and also inverse $(r=-0.066, p<0.01)$, refuting the strength of relationship between body mass and foot posture.

BMI cut-points and percentiles were used to define underweight, normal weight, overweight and obese. Within the total 3217 children, $142(4.4 \%)$ were underweight, 2407 (74.8\%) were normal weight, 469 (16.1\%) were overweight and $199(6.2 \%)$ were obese (figure 4$)$.

Combining categories, 668 (20.8\%) of all children were overweight or obese.

The foot posture categories were analysed for distribution across the BMI categories (table 5). The largest overlap of FPI and BMI categories were normal weight/ normal FPI range, $\mathrm{n}=1325$ (41.2\%); normal weight/ pronated foot posture, $\mathrm{n}=728(22.6 \%)$; overweight or obese/normal foot posture, $\mathrm{n}=383(11.9 \%)$. Supinated

Table 1 Descriptive statistics for the amalgamated datasets

\begin{tabular}{lllllrl}
\hline Variable & N & Range & Minimum & Maximum & Mean & SD \\
\hline Age (years) & 3217 & 12 & 3 & 15 & 8.67 & 2.02 \\
BMI $\left(\mathrm{kg} / \mathrm{m}^{2}\right)$ & 3217 & 28.57 & 10.57 & 39.14 & 19.08 & 4.05 \\
FPI right & 3217 & 16 & -4 & 12 & 4.20 & 3.00 \\
FPI left & 3217 & 16 & -4 & 12 & 4.11 & 2.92 \\
\hline
\end{tabular}

Due to variation between the constituent datasets, main results emerged from the variables of age, BMI and FPI left. Gender ratio was 1699 males: 1518 females.

BMI, body mass index; PFPI, foot posture index. 
Table 2 The FPI range which refer to foot posture categories were collated for each year of age

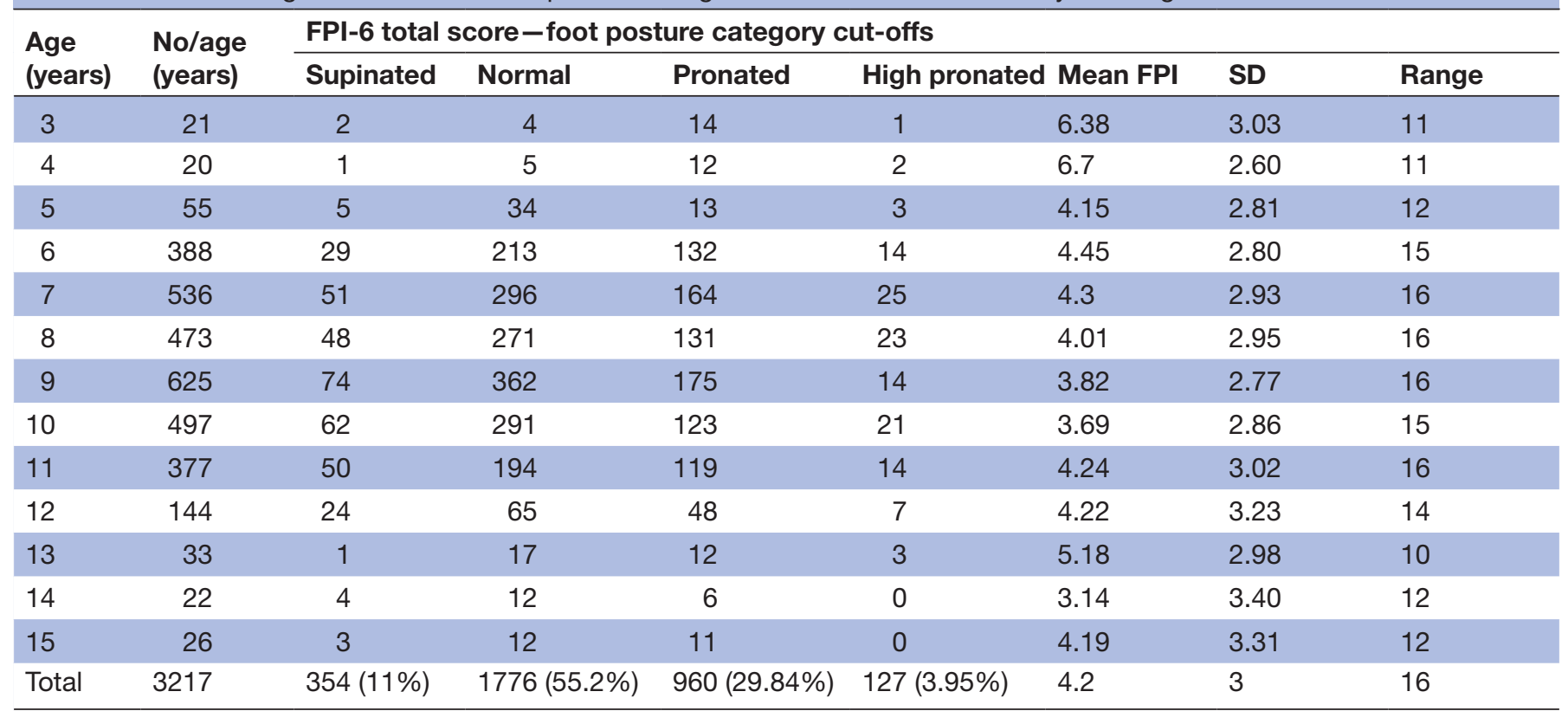

The median FPI total score across the study population was 4 (3) points, with the trend of reduced FPI with increased age confirmed. FPI, foot posture index.

feet across all BMI categories returned $\mathrm{n}=354(11.0 \%)$. Exploring the association between the pronated foot and BMI across FPI ranges showed that $960(29.8 \%)$ children had foot posture that was pronated, FPI $>6$. Of these, $44(4.5 \%)$ were underweight, $728(75.8 \%)$ were normal weight, 188 were overweight or obese $(19.5 \%)$. Further, 127 children had highly pronated foot posture, FPI $>10$. Of these, $12(9.4 \%)$ were underweight, $94(74.0 \%)$ were normal weight and $21(16.5 \%)$ were overweight or obese.

\section{DISCUSSION}

This study is the largest investigation to explore paediatric foot posture using the FPI, paediatric anthropometry using BMI and to analyse the regularly cited influence of increased body weight as a potentiating factor for flatfeet across childhood. This investigation of paediatric foot posture includes children aged from 3 to 15 years, superseding previous, smaller or age-limited, studies. ${ }^{26} 27$

This study confirms the pronated foot as the common foot posture of childhood, with mean FPI of +4 , and 3-point SD, such that average normal FPI range for children aged $3-15$ years was between the FPI range +1 to +7 $($ mean $\pm S D)$. We found that the mean FPI scores reduced

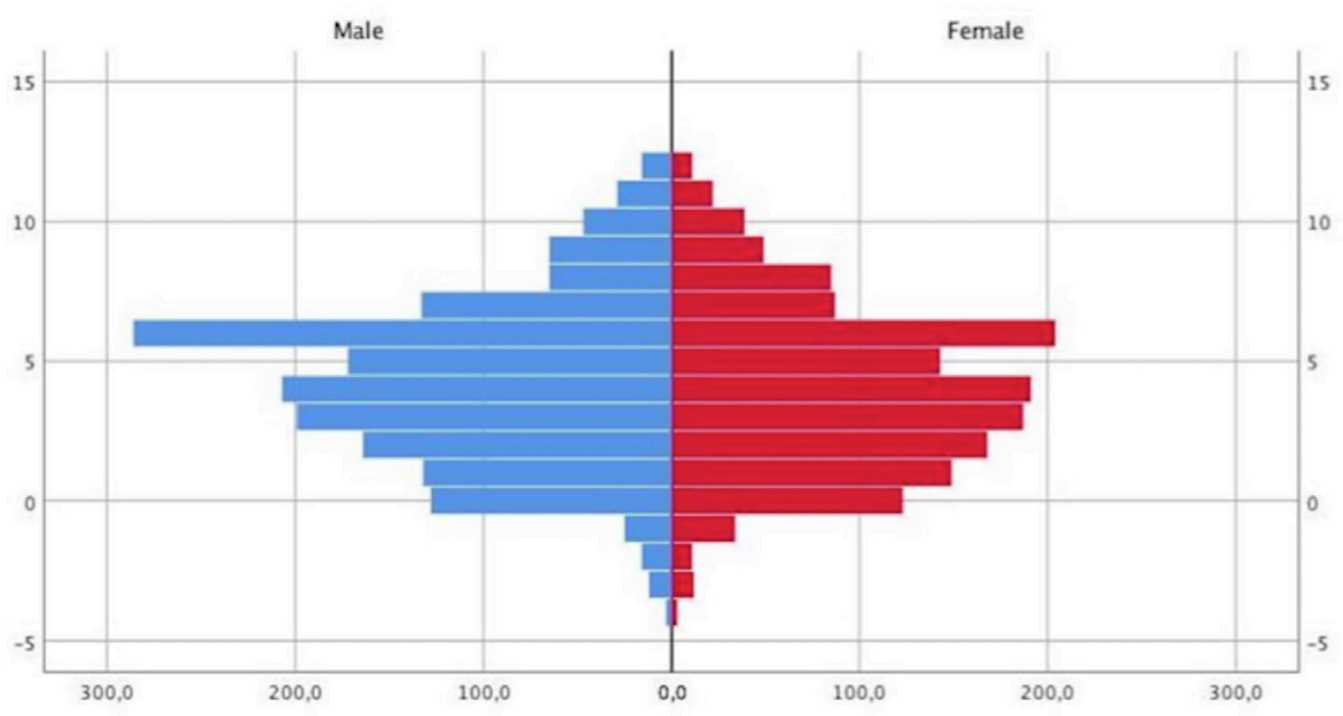

Figure 2 FPI frequency plot for gender. FPI, foot posture index. 
Table 3 FPI total scores versus age year groups

\begin{tabular}{|c|c|c|c|c|c|c|c|c|c|c|c|c|c|c|c|c|c|c|}
\hline $\begin{array}{l}\text { FPI/age } \\
\text { (years) }\end{array}$ & -4 & -3 & -2 & -1 & 0 & 1 & 2 & 3 & 4 & 5 & 6 & 7 & 8 & 9 & 10 & 11 & 12 & Total $/ \mathbf{n}$ \\
\hline 3 & 0 & 0 & 0 & 1 & 1 & 0 & 0 & 1 & 2 & 1 & 2 & 0 & 3 & 4 & 4 & 0 & 2 & 21 \\
\hline 4 & 0 & 0 & 0 & 0 & 1 & 0 & 0 & 0 & 3 & 3 & 5 & 2 & 2 & 1 & 1 & 2 & 0 & 20 \\
\hline 5 & 0 & 0 & 1 & 0 & 6 & 3 & 7 & 11 & 7 & 3 & 6 & 2 & 2 & 1 & 1 & 4 & 1 & 55 \\
\hline 6 & 0 & 1 & 3 & 6 & 24 & 31 & 45 & 46 & 47 & 38 & 69 & 30 & 19 & 11 & 6 & 8 & 4 & 388 \\
\hline 7 & 1 & 6 & 4 & 3 & 39 & 43 & 48 & 72 & 69 & 55 & 90 & 33 & 22 & 27 & 14 & 7 & 3 & 536 \\
\hline 9 & 3 & 3 & 5 & 11 & 55 & 61 & 60 & 72 & 81 & 78 & 92 & 48 & 21 & 14 & 9 & 8 & 4 & 625 \\
\hline 10 & 0 & 5 & 3 & 14 & 41 & 59 & 68 & 71 & 54 & 39 & 61 & 23 & 17 & 12 & 17 & 9 & 4 & 497 \\
\hline 11 & 1 & 3 & 4 & 10 & 29 & 22 & 31 & 48 & 58 & 32 & 52 & 29 & 26 & 14 & 10 & 3 & 5 & 377 \\
\hline 12 & 0 & 2 & 2 & 6 & 17 & 9 & 12 & 7 & 19 & 11 & 19 & 14 & 12 & 6 & 5 & 3 & 0 & 144 \\
\hline 13 & 0 & 0 & 0 & 0 & 1 & 2 & 1 & 6 & 2 & 5 & 2 & 7 & 2 & 2 & 3 & 0 & 0 & 33 \\
\hline$\%$ & 0.2 & 0.7 & 0.8 & 1.8 & 7.8 & 8.7 & 10.3 & 12.0 & 12.4 & 9.8 & 15.2 & 6.8 & 4.7 & 3.5 & 2.7 & 1.6 & 0.8 & 100 \\
\hline
\end{tabular}

\%Percentage. FPI, foot posture index; R, range.

with age, in non-linear pattern, and within a wide range. The SD approximated $75 \%$ of the FPI mean at every age, confirming the considerable and normal variation in foot posture across childhood.

The greatest number of children across all ages displayed FPI within the 0 to +5 FPI range, that is, normal foot posture. Next common were children with pronated feet. The least common FPI categories were either supinated or highly pronated, indicative of the foot types that should arrest the attention of clinicians, as less usual presentations. Flatfoot or pronated foot posture was generally found to decline with age, but mean reduction was non-linear and modest, from +6 at age 3 years to +3 at 14 years. Importantly, the normal FPI range of variation was broad: -1 to +11 at age 3 years, and FPI +3 to +9 at age 14 years.

The relationship between increased BMI and flatfeet is again refuted by the findings of this study which found that only $16.5 \%$ of the children with highly pronated feet, were also overweight or obese. Our results contrasted with many older studies which asserted that heavier, fatter children have flatter feet.

\begin{tabular}{|c|c|c|c|c|c|c|c|c|c|c|}
\hline $\begin{array}{l}\text { Age } \\
\text { (years) }\end{array}$ & $-2 S D$ & $-1 S D$ & Mean & +1 SD & +2 SD & $\begin{array}{l}\text { Sample } \\
\text { size }\end{array}$ & SD & $\begin{array}{l}\text { FPI median } \\
( \pm 1 \text { SD) }\end{array}$ & $\begin{array}{l}\text { FPI median } \\
( \pm 2 S D)\end{array}$ & $\begin{array}{l}\text { Clinical alert } \\
\text { FPI > } 2 \text { SD }\end{array}$ \\
\hline 3 & 0.02 & 3.60 & 7.19 & 10.78 & 12.78 & 21 & 3.6 & $8(4-12)$ & $8(0-12)$ & $<0$ or $>12$ \\
\hline 4 & 1.11 & 3.76 & 6.40 & 9.04 & 11.79 & 20 & 2.6 & $6(3-9)$ & $6(0-12)$ & $<0$ or $>12$ \\
\hline 5 & -2.41 & 0.91 & 4.22 & 7.54 & 10.68 & 55 & 3.3 & $3(0-6)$ & $3(-3$ to 9$)$ & $<-3$ or $>9$ \\
\hline 6 & -1.37 & 1.50 & 4.36 & 7.22 & 10.71 & 388 & 2.9 & $4(1-7)$ & $4(-2$ to 10$)$ & $<-2$ or $>10$ \\
\hline 7 & -1.53 & 1.40 & 4.32 & 7.24 & 10.67 & 536 & 2.9 & $4(1-7)$ & $4(-2$ to 10$)$ & $<-2$ or $>10$ \\
\hline 8 & -1.64 & 1.33 & 4.29 & 7.25 & 10.15 & 473 & 2.9 & $4(1-7)$ & $4(-1$ to 10$)$ & $<-1$ or $>10$ \\
\hline 9 & -1.74 & 1.11 & 3.96 & 6.81 & 9.83 & 625 & 2.8 & $4(1-7)$ & $4(-1$ to 10$)$ & $<-1$ or $>10$ \\
\hline 10 & -2.31 & 0.73 & 3.77 & 6.81 & 9.92 & 497 & 3.0 & $3(0-6)$ & $4(-3$ to 9$)$ & $<-3$ or $>9$ \\
\hline 11 & -1.77 & 1.28 & 4.33 & 7.38 & 10.20 & 377 & 3.0 & $4(1-7)$ & $4(-2$ to 10$)$ & $<-2$ or $>10$ \\
\hline 12 & -2.43 & 0.92 & 4.26 & 7.61 & 10.73 & 144 & 3.3 & $4(1-7)$ & $4(-2$ to 10$)$ & $<-2$ or $>10$ \\
\hline 13 & -0.03 & 2.71 & 5.45 & 8.19 & 11.18 & 33 & 2.7 & $5(2-8)$ & 5 (-1 to 11$)$ & $<-1$ or $>11$ \\
\hline 14 & -3.89 & -0.28 & 3.32 & 6.92 & 9.92 & 22 & 3.6 & $4(1-7)$ & 4 (-2 to 9$)$ & $<-2$ or $>9$ \\
\hline 15 & -2.84 & 0.77 & 4.38 & 7.99 & 11.18 & 26 & 3.6 & $4(0-8)$ & $4(-4$ to 12$)$ & $<-4$ or $>12$ \\
\hline
\end{tabular}

FPI, foot posture index. 


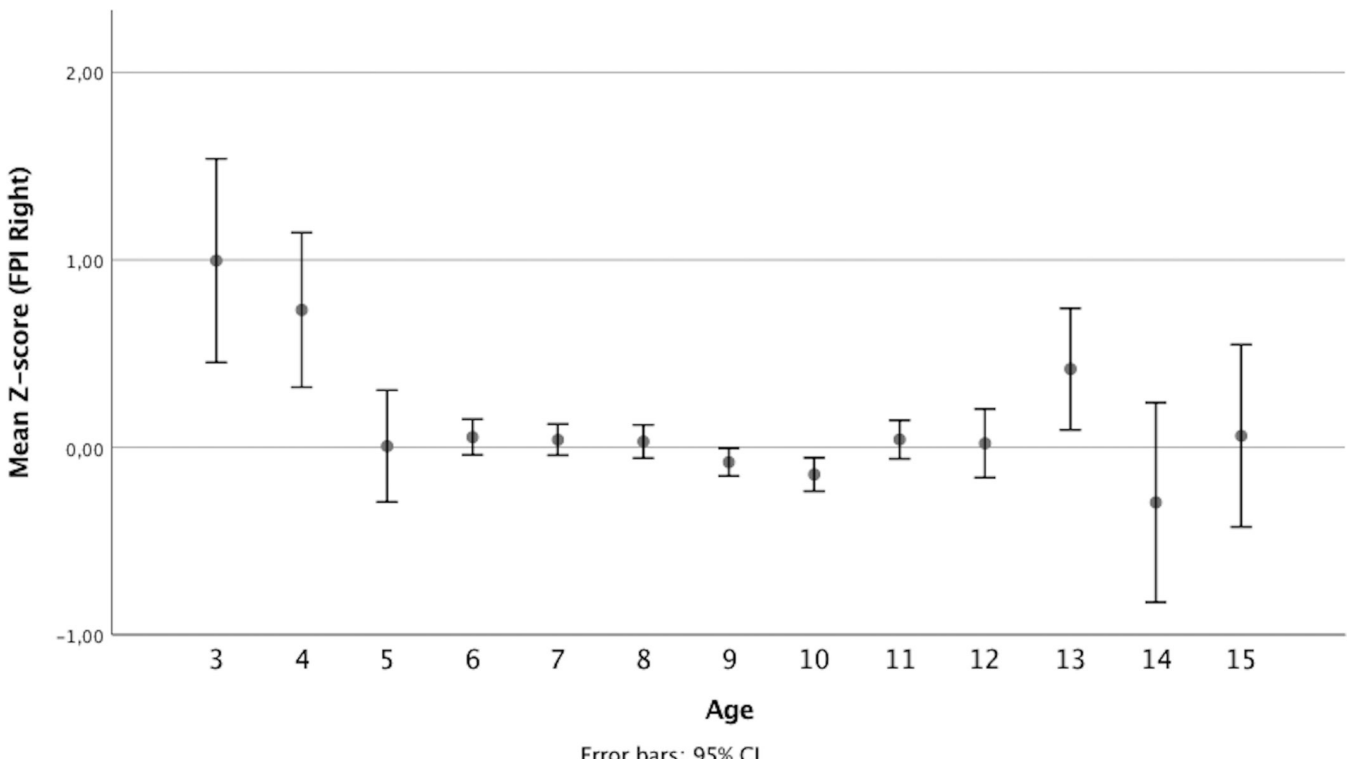

Figure 3 Simple error bars display the relationship between FPI and age. FPI, foot posture index.

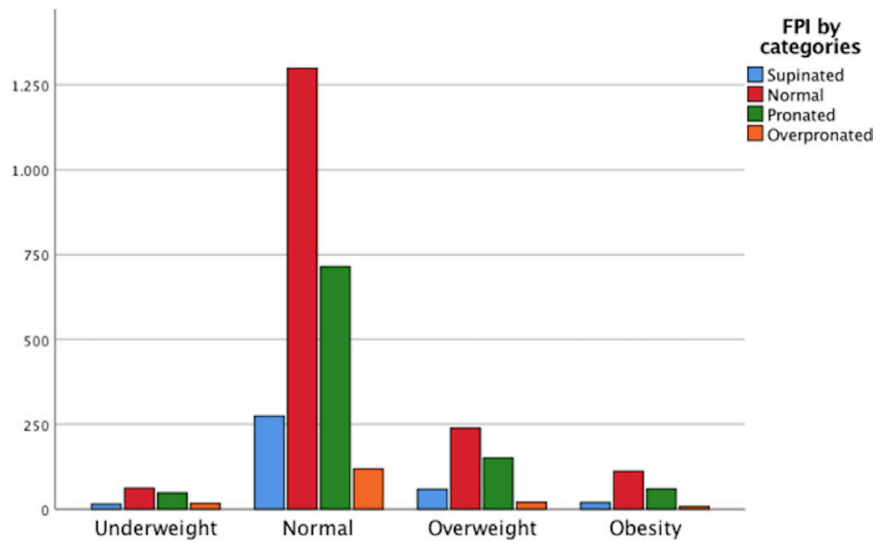

Figure 4 Frequency plot of BMI category versus FPI category. BMI, body mass index; FPI, foot posture index.
Importantly, the previous studies all assessed foot posture using a footprint-based method of foot posture assessment, ${ }^{59}{ }^{40}$ a method which may well represent adipose tissue spread with weight bearing, rather than anatomical foot morphology, more directly evaluated using the FPI. ${ }^{25} 2641$ While it is concerning to find that $21 \%$ of the children in this study were overweight or obese, association with flatfeet is not found.

The availability of average FPI whole scores enables clinicians to inform parents as to what is 'average' and what is 'normal' at any age, as statistically defined. The availability of FPI scores within 1 SD above and below the mean, enables clinicians to confirm for parents that their child approximates with two-thirds, or $68 \%$, of children for a specific age. Further, FPI scores within 2 SD above and below the mean, enable clinicians to inform parents

Table 5 FPI and BMI category distributions showing greatest concordance between normal FPI and normal BMI

\begin{tabular}{|c|c|c|c|c|c|}
\hline & & Underweight & Normal & $\begin{array}{l}\text { Overweight or } \\
\text { obese }\end{array}$ & Total \\
\hline Supinated & Count & $18^{a}$ & $260^{a}$ & $76^{a}$ & 354 \\
\hline \multirow[t]{2}{*}{ Normal } & Count & $68^{a}$ & $1325^{a}$ & $383^{a}$ & 1776 \\
\hline & Expected count & 78.4 & 1328.8 & 368.8 & 1776 \\
\hline \multirow[t]{2}{*}{ Highly pronated } & Count & $12^{\mathrm{a}}$ & $94^{\mathrm{b}}$ & $21^{b}$ & 127 \\
\hline & Expected count & 5.6 & 95 & 26.4 & 127 \\
\hline Total & Count & 142 & 2407 & 668 & 3217 \\
\hline
\end{tabular}

Superscript letters denote a subset of FPI (left) categories whose column proportions did not differ significantly from each other at the level of 0.05 .

BMI, body mass index; FPI, foot posture index. 
that their child is within the normal 95\% range, approximating one-quarter, or $27 \%$, of same age children. Such reference data help appreciation of the range of "normal range' for foot posture, similar to that for the onset of independent walking (age range of 10-16 months, mean age approximating 12-13 months). ${ }^{18}$

The focus of this investigation has been to better elucidate the normal range of foot posture across childhood, as is commonly assessed by many clinicians using the FPI. Simultaneously, the authors aimed to provide clinicians with a robust reference guide of normal values within statistical bounds. The culmination of this aim is provided as a reference table (table 4). The scale of this investigation renders its findings stronger than those of the 1000 Norms protocol for most age (years) groups, as the comparative number of participants reveals. The 1000 Norms protocol will include ages 3-9 years, 140 (20 per age year); ages $10-19$ years, 160 (16 per age year). ${ }^{42}$ By comparison, this study informs for: ages 3-9 years, 2796 (20-764 per age year); ages 10-15 years, 1304 (1-634 per age year).

Clinicians must move beyond flatfoot posture appearance as an indicator for intervention, and instead appreciate the range of normal variation, and only respond to more pertinent factors as outlined by the 3 quick questions screening tool (addressing pain presentations, left vs right limb symmetry, paediatric age range) ${ }^{20}$ Clinicians need to appreciate the normal range of many developmental features, yet simultaneously be alert to the level at which clinical concern should be raised. The tabulated reference guide provided from this study, will be of immediate clinical relevance.

The striking finding of this study is not that paediatric flexible flatfoot is largely normal, it is that the supinated paediatric foot is far more likely to be abnormal, especially at age 3 and 4 years. An FPI of -2 or less, must be considered 'abnormal' until shown otherwise, as it is outside normal range at any age, and should prompt neurological assessment.

Limitations of this investigation include the cross-sectional nature of the design and the ethnicity of participants (largely Caucasian). Further, the sample does not evenly represent children from each country, nor each year of age; hence, caution is indicated for ages 3-7 and 14-15 years where sampling was least. In addition, consideration that this study is a collation of smaller discrete studies, and should be consider measurement errors, although all followed the same protocol. ${ }^{3}$ Prospective data avail stronger evidence of foot posture change over time. $^{4}$

\section{CONCLUSIONS}

This is the largest study of paediatric foot posture to date. Importantly, the main finding is to denounce the paediatric flatfoot as deviant. This study confirms that the 'flat' or pronated foot is the common foot posture of childhood, with FPI score of +4 (3) the average finding.
A wide normal range of foot posture across childhood is confirmed (16 FPI points, ie, -2 to +12 ).

The reference data produced from the findings of this study will assist clinicians in standardised decision-making.

Increased paediatric BMI was not associated with flatter feet, questioning the validity of footprint-derived measures.

Acknowledgements The authors would like to express their gratitude to the University of Malaga for their support through Plan Propio de Investigacion and Sergio López Casares, manager of projects of the University of Extremadura.

Contributors GG-N, AM-N and AME contributed to the conception of this study. GG-N, AM-N and AME did the statistical analysis. Data collectors were collected in Spain JM-A and PA-G and in Australia AME. GG-N, AM-N, PA-G, JM-A and AME were involved in the writing and in the review of the manuscript.

Funding The University of Malaga through of Plan Propio de Investigacion Competing interests None declared.

Patient consent for publication Parental/guardian consent obtained.

Ethics approval All procedures performed in studies involving human participants were in accordance with the ethical standards of the institutional and/or national research committee and with the 1964 Helsinki declaration and its later amendments or comparable ethical standards (Universities of Malaga CEUMA 91/2016H, Extremadura ID 59/2012, University of Wales Institute, Cardiff and University of South Australia Charles Sturt University Ethics Committees approval number 10/291 and 89/2012).

Provenance and peer review Not commissioned; externally peer reviewed.

Data sharing statement Available data can be obtained by contacting the corresponding author.

Open access This is an open access article distributed in accordance with the Creative Commons Attribution Non Commercial (CC BY-NC 4.0) license, which permits others to distribute, remix, adapt, build upon this work non-commercially, and license their derivative works on different terms, provided the original work is properly cited, appropriate credit is given, any changes made indicated, and the use is non-commercial. See: http://creativecommons.org/licenses/by-nc/4.0/.

\section{REFERENCES}

1. Carli A, Saran N, Kruijt J, et al. Physiological referrals for paediatric musculoskeletal complaints: A costly problem that needs to be addressed. Paediatr Child Health 2012;17:e93-7.

2. Pfeiffer M, Kotz R, Ledl T, et al. Prevalence of flat foot in preschoolaged children. Pediatrics 2006;118:634-9.

3. Drefus LC, Kedem P, Mangan SM, et al. Reliability of the Arch Height Index as a Measure of Foot Structure in Children. Pediatr Phys Ther 2017;29:83-8.

4. Redmond AC, Crosbie J, Ouvrier RA. Development and validation of a novel rating system for scoring standing foot posture: the foot posture index. Clin Biomech 2006;21:89-98.

5. Chen KC, Yeh CJ, Kuo JF, et al. Footprint analysis of flatfoot in preschool-aged children. Eur J Pediatr 2011;170:611-7.

6. Chang $\mathrm{CH}$, Chen YC, Yang WT, et al. Flatfoot diagnosis by a unique bimodal distribution of footprint index in children. PLoS One 2014;9:e115808.

7. Nemeth B. The diagnosis and management of common childhood orthopedic disorders. Curr Probl Pediatr Adolesc Health Care 2011;41:2-28.

8. Uden H, Scharfbillig R, Causby $\mathrm{R}$. The typically developing paediatric foot: How flat should it be? A systematic review. J Foot Ankle Res $2017 ; 10$.

9. Sadeghi-Demneh E, Azadinia F, Jafarian F, et al. Flatfoot and obesity in school-age children: a cross-sectional study. Clin Obes 2016;6:42-50.

10. Evans AM, Rome K, Peet L. The foot posture index, ankle lunge test, Beighton scale and the lower limb assessment score in healthy children: a reliability study. J Foot Ankle Res 2012;5:1.

11. Martínez-Nova A, Gijón-Noguerón G, Alfageme-García P, et al. Foot posture development in children aged 5 to11 years: A three-year prospective study. Gait Posture 2018;62:280-4.

12. Mickle KJ, Steele JR, Munro BJ. Is the foot structure of preschool children moderated by gender? J Pediatr Orthop 2008;28:593-6.

13. Stolzman S, Irby MB, Callahan $A B$, et al. Pes planus and paediatric obesity: a systematic review of the literature. Clin Obes 2015;5:52-9. 
14. Kothari A, Dixon PC, Stebbins J, et al. The relationship between quality of life and foot function in children with flexible flatfeet. Gait Posture 2015;41:786-90.

15. Lin CJ, Lai KA, Kuan TS, et al. Correlating factors and clinical significance of flexible flatfoot in preschool children. $J$ Pediatr Orthop 2001;21:378-82.

16. Labovitz JM. The algorithmic approach to pediatric flexible pes planovalgus. Clin Podiatr Med Surg 2006;23:57-76.

17. Evans AM. The flat-footed child -- to treat or not to treat: what is the clinician to do? J Am Podiatr Med Assoc 2008;98:386-93.

18. Evans A. Pocket podiatry. Paediatrics 2010.

19. Gijon-Nogueron G, Sanchez-Rodriguez R, Lopezosa-Reca E, et al. Normal values of the Foot Posture Index in a young adult Spanish population: a cross-sectional study. J Am Podiatr Med Assoc 2015;105:42-6.

20. Evans AM. Mitigating clinician and community concerns about children's flatfeet, intoeing gait, knock knees or bow legs. J Paediatr Child Health 2017;53:1050-3.

21. Evans AM, Rome K. A Cochrane review of the evidence for nonsurgical interventions for flexible pediatric flat feet. Eur J Phys Rehabil Med 2011;47:69-89.

22. Rome K, Ashford RL, Evans A. Non-surgical interventions for paediatric pes planus. Cochrane Database Syst Rev 2010:CD006311.

23. Kane K. Foot orthoses for pediatric flexible flatfoot: evidence and current practices among Canadian physical therapists. Pediatr Phys Ther 2015;27:53-9.

24. Harris EJ. The natural history and pathophysiology of flexible flatfoot. Clin Podiatr Med Surg 2010;27:1-23.

25 Redmond AC, Crane YZ, Menz HB. Normative values for the Foot Posture Index. J Foot Ankle Res 2008;1:6.

26. Evans AM, Karimi L. The relationship between paediatric foot posture and body mass index: do heavier children really have flatter feet? J Foot Ankle Res 2015;8:46.

27. Gijon-Nogueron G, Montes-Alguacil J, Alfageme-Garcia P, et al. Establishing normative foot posture index values for the paediatric population: a cross-sectional study. J Foot Ankle Res 2016;9:24

28. Morrison SC, Ferrari J. Inter-rater reliability of the Foot Posture Index (FPI-6) in the assessment of the paediatric foot. J Foot Ankle Res 2009;2:26.
29. Banwell HA, Paris ME, Mackintosh S, et al. Paediatric flexible flat foot: how are we measuring it and are we getting it right? A systematic review. J Foot Ankle Res 2018;11.

30. Evans AM. The paediatric flat foot and general anthropometry in 140 Australian school children aged 7 - 10 years. J Foot Ankle Res 2011;4:12.

31. Targett R, Mathieson I. Evaluation of foot posture development in children between three and eleven years of age using the foot posture index. J Foot Ankle Res 2010;3:P17.

32. Scharfbillig RW, Jones S, Scutter S. Sever's disease-Does it effect quality of life? Foot 2009;19:36-43.

33. Williams CM, Tinley $\mathrm{P}$, Curtin $\mathrm{M}$, et al. Hand preference in children with an idiopathic toe walking gait. Acta Paediatr 2012;101:e14 0-e141.

34. Keenan $\mathrm{AM}$, Redmond $\mathrm{AC}$, Horton $\mathrm{M}$, et al. The Foot Posture Index: Rasch analysis of a novel, foot-specific outcome measure. Arch Phys Med Rehabil 2007;88:88-93.

35. Sobradillo B, Aguirre A, Aresti U, et al. Curvas y tablas de crecimiento (estudio longitudinal y transversal). Fund: Faustino Orbegozo Eizaguirre, 2004. http://www.aepap.org/pdf/f_orbegozo_ 04.pdf

36. Australian Bureau of Statistics. Australian Health Survey: First Results, 2011-12. CAT no 4364055003. 2012:1-61.

37. Noo, Ncmp. National Child Measurement Programme. Changes in children' s body mass index. 2013:1-36 http://www.noo.org.uk/ uploads/doc/vid 17929 NCMP Changes children.pdf

38. Menz HB. Analysis of paired data in physical therapy research: time to stop double-dipping? J Orthop Sports Phys Ther 2005;35:477-8.

39. Mickle KJ, Steele JR, Munro BJ. The feet of overweight and obese young children: are they flat or fat? Obesity 2006;14:1949-53.

40. Villarroya MA, Esquivel JM, Tomás C, et al. Assessment of the media longitudinal arch in children and adolescents with obesity: footprints and radiographic study. Eur J Pediatr 2009;168:559-67.

41. Gijon-Nogueron G, Montes-Alguacil J, Martinez-Nova A, et al. Overweight, obesity and foot posture in children: a cross-sectional study. J Paediatr Child Health 2017;53:33-7.

42. McKay MJ, Baldwin JN, Ferreira P, et al. 1000 Norms Project: protocol of a cross-sectional study cataloging human variation. Physiotherapy 2016;102:50-6. 